\title{
Supporting information: Bet-hedging strategies in expanding populations
}

\author{
Paula Villa Martín \\ Biological Complexity Unit, Okinawa Institute of Science and \\ Technology Graduate University, Onna, Okinawa 904-0495, Japan \\ Miguel A. Muñoz \\ Departamento de Electromagnetismo y Fúsica de la Materia and Instituto Carlos I de Fúsica Teórica y Computacional, \\ Facultad de Ciencias, Universidad de Granada, 18071 Granada, Spain \\ Simone Pigolotti* \\ Biological Complexity Unit, Okinawa Institute of Science and \\ Technology Graduate University, Onna, Okinawa 904-0495, Japan. \\ (Dated: April 2, 2019)
}

\section{NUMERICAL INTEGRATION OF THE STOCHASTIC FISHER EQUATION}

In this section we describe in details the methods for the integration of the wave equations of the two-phenotype model used for simulations in the Main Text.

\section{Fisher wave}

We consider the Fisher equation

$$
\dot{f}(x, t)=D \nabla^{2} f(x, t)+\sigma(x, t) f(x, t)(1-f(x, t)),
$$

where $f(x, t)$ is the population density at space $x$ and time $t$, and $\sigma(x, t)$ is the local growth rate.

We employ a finite-difference fourth-order Runge-Kutta method. The systems is initialized by fixing $f\left(x_{i}, 0\right)=1$ for $i \in(0,50)$ and $f(x, t)=0$ for $i>50$. The spatial mesh $d x$ is fixed by means of an adaptive routine. We intialize the routine with an initial guess $d x=0.14$. Then

1. We let the system evolve until the front reaches a stationary state.

2. We compute the smallest values of $x$ for which $f(x, t)>\theta$ for $\theta=3 / 4$ and $\theta=1 / 4$. We denote these two values as $x_{3 / 4}$ and $x_{1 / 4}$ respectively.

3. We measure the precisions $\Delta f_{3 / 4}=f\left(x_{3 / 4}-d x\right)-f\left(x_{3 / 4}\right), \Delta f_{1 / 4}=f\left(x_{1 / 4}-d x\right)-f\left(x_{1 / 4}\right)$.

4. If $\Delta f_{3 / 4}>0.01$ and $\Delta f_{1 / 4}>0.01$, then $d x$ is accepted as a valid increment.

5. Otherwise, the system is reset to the initial condition and the routine is again run for $d x=d \tilde{x}-0.01$; being $d \tilde{x}$ the previous spatial mesh.

Once $d x$ is determined, $d t$ is fixed following the Courant-Friedrichs-Lewy condition for an explicit integration method $[1]:$

$$
\frac{v_{\max } d t}{d x} \leq 1
$$

\footnotetext{
* simone.pigolotti@oist.jp
} 
being $v_{\max }$ the estimated maximum velocity of the wave. We fix $v_{\max }=100$, which is sufficiently large compared to velocities in our simulations.

Temporal environmental switch is numerically implemented with a simple first-order algorithm. At the beginning of each time step, the state of environment is switched with probability $k d t$. We verified that this quantity is always sufficiently small, so that the first-order algorithm yields reliable results. A similar algorithm is implemented for spatial environmental variations to sequentially assign an environmental state to each lattice site.

\section{Stochastic Fisher wave}

We consider the stochastic Fisher equation [2]

$$
\dot{f}(x, t)=D \nabla^{2} f+\sigma(t) f(1-f)+\sqrt{\frac{2}{N} f(1-f)} \xi(x, t)
$$

where $\xi(x, t)$ a Gaussian white noise satisfying $\langle\xi(x, t)\rangle=0$ and $\left\langle\xi(x, t) \xi\left(x^{\prime}, t^{\prime}\right)\right\rangle=\delta\left(x-x^{\prime}\right) \delta\left(t-t^{\prime}\right)$. For later convenience, we define the noise amplitude $\gamma(t) \equiv \sqrt{\frac{2}{N} f(t)(1-f(t))}$.

Numerical integration in the presence of noise is subtle. In particular, one has to figure out how to deal with the unphysical values $f(x, t)<0$ and $f(x, t)>1$ that can result due to finite numerical precision. Depending on parameter range, the naive replacement $f(x, t)=0$ or $f(x, t)=1$ when $f(x, t)<0$ and $f(x, t)>1$, respectively, may introduce a substantial bias. In particular, an incorrect integration of $f(x, t)$ at the front, where $f(x, t)$ is small, might lead to an large error in the estimated velocity. However, when $f(x, t)$ is small so that $\gamma(t) \simeq \sqrt{\frac{2}{N} f(t)}$, this problem can be circumvented by integrating the noise term exactly [3], while integrating the rest of the equation deterministically [4].
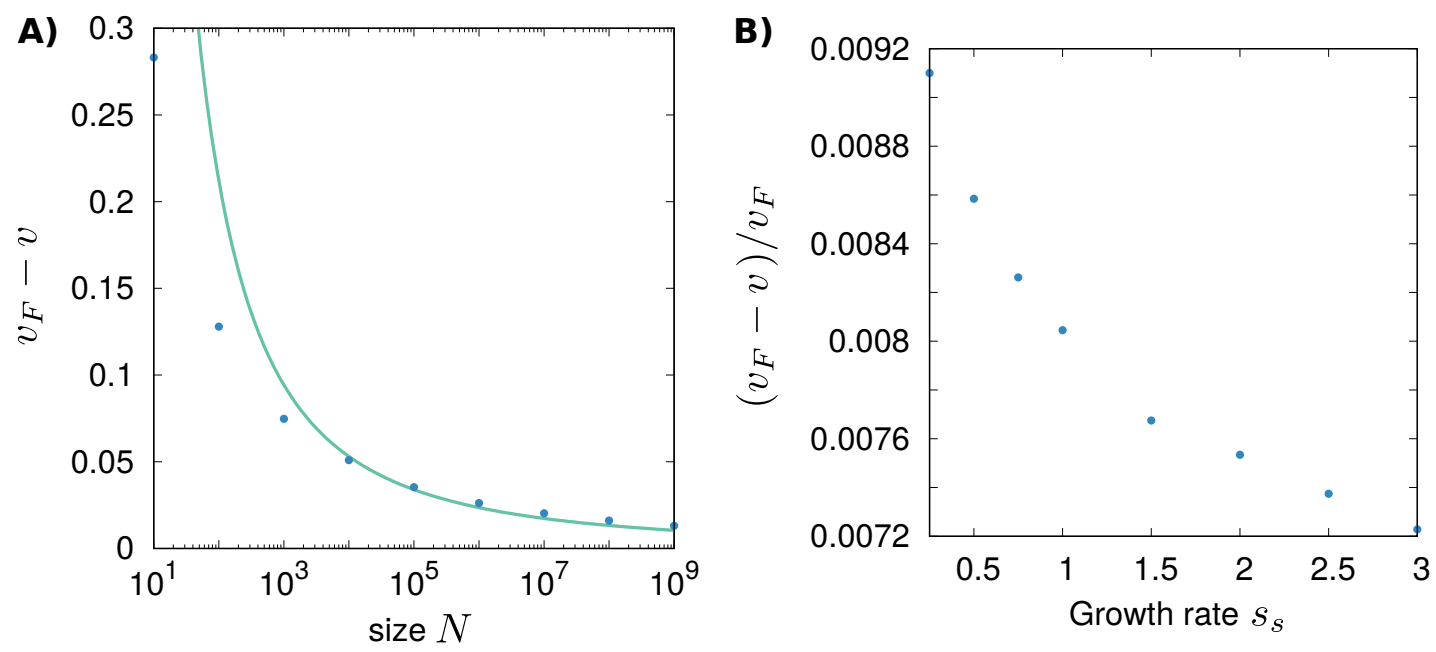

FIG. 1. Size scaling and maximum error estimation for our integration method. Panel A) shows the curve $4.5(\log N)^{-2}$ and the difference $v_{F}-v$ for $k=0, \alpha=0, s_{s}=1$, and different system sizes. Panel B) shows $\left(v_{F}-v\right) / v_{F}$ for $N=10^{9}$ and different growth rates employed in this work. These results suggest that our integration method is precise and have a maximum error of $0.0018 \%$.

We follow this strategy and integrate the equation mixing two different algorithms, depending on the local value of $f(x, t)$ :

- If $f(x, t)>\theta$ : we employ the Milstein method (order 1). Defining $\beta(t) \equiv D \nabla^{2} f(t)+\sigma(t) f(t)(1-f(t))$, the local field is updated according to the rule

$$
f(x, t+d t)=f(x, t)+\beta(t) d t+\gamma(t) \Delta+\frac{1}{2} \gamma(t) \frac{\partial \gamma}{\partial f(t)}\left(\Delta^{2}-d t\right)
$$


being $\Delta=\sqrt{d t} g(t)$, where $g(t)$ is a Gaussian random variable with zero mean and unit variance, drawn independently at each time step.

- If $f(x, t)<\theta$ we employ a split-step numerical integration:

1. Non-linear and diffusion terms. The term $\dot{f}(x, t)=D \nabla^{2} f-\sigma(t) f^{2}$ is integrated by means of the RungeKutta method obtaining a first solution $f^{*}$.

2. Linear and stochastic terms. The term $\sigma(t) f+\sqrt{\frac{2}{N} f} \xi(x, t)$ is integrated in an exact way, see [3]:

$$
f(x, t)=r_{\text {Gamma }}\left\{r_{\text {Poisson }}\left\{\lambda f^{*}(x, t) e^{\sigma(t) t}\right\}\right\} / \lambda .
$$

being $\lambda=2 \sigma(t) /\left[\gamma^{2} e^{\sigma(t) t}\right]$, and $r_{\text {Gamma }}, r_{\text {Poisson }}$ random variables from Gamma and Poisson probability distributions respectively, i.e. $\operatorname{Prob}\left[r_{\mathrm{Gamma}}(a)=z\right]=z^{a-1} e^{-z} / \Gamma[a]$ and $\operatorname{Prob}\left[r_{\mathrm{Poisson}}(a)=z\right]=a^{z} e^{-a} / z !$.

To check the precision of our method we integrated the stochastic equation (3) for $k=0, \alpha=0$, and different growth rates $s_{s}$ and compared the results to the analytical Fisher velocity $v_{F}=2 \sqrt{D s_{s}}$. For large population size $N$, the velocity $v$ of the wave asymptotically goes as $v_{F}-v \simeq C \ln ^{-2}(N)$ [5]. Our numerical integration is consistent with this asymptotic relation from $N \simeq 10^{4}$ (figure $1 \mathrm{~A}$ ) with a root-mean-square deviation of 0.002 . We have also obtained the values $\left(v_{F}-v\right) / v_{F}$ for the different growth rates employed in this work to obtain an estimation of the maximum error (see figure $1 \mathrm{~B}$ ). Note that $v_{F}$ is not the actual velocity of the finite system, so the relative error $\left(v_{F}-v\right) / v_{F}$ is, in fact, smaller. The maximum error is around $0.9 \%$, that, considering the results of figure $\left.1 \mathrm{~A}\right)$ leads to an overestimated error of about $0.0018 \%$.

[1] Richard Courant, Kurt Friedrichs, and Hans Lewy, "On the partial difference equations of mathematical physics," IBM journal of Research and Development 11, 215-234 (1967).

[2] Kirill S Korolev, Mikkel Avlund, Oskar Hallatschek, and David R Nelson, "Genetic demixing and evolution in linear stepping stone models," Reviews of modern physics 82, 1691 (2010).

[3] Ivan Dornic, Hugues Chaté, and Miguel A Munoz, "Integration of langevin equations with multiplicative noise and the viability of field theories for absorbing phase transitions," Physical review letters 94, 100601 (2005).

[4] Haim Weissmann, Nadav M Shnerb, and David A Kessler, "Simulation of spatial systems with demographic noise," Physical Review E 98, 022131 (2018).

[5] Éric Brunet and Bernard Derrida, "Effect of microscopic noise on front propagation," Journal of Statistical Physics 103, 269-282 (2001). 